\title{
Research on technology of target location and tracking based on Internet of things
}

\author{
Jie Zhang \\ School of Electrical Engineering, Chengdu Textile College, Chengdu, China \\ 3047699076@qq.com
}

Keywords: Positioning; Tracking; Sensor; Internet of things

\begin{abstract}
With the development of networking technology, targeting and tracking technology has been improved and enhanced. Difficult to capture the movement of the target, which brings great difficulty to locate and track algorithm exists for the current low efficiency, high energy consumption and other issues, this study positioning accuracy, tracking accuracy rate, energy consumption for a study evaluation. Through the current location and tracking algorithms for analysis, we propose a space-based division of Things targeting and tracking algorithm, this method can effectively improve the accuracy of target tracking, reduce energy consumption, has some practical value.
\end{abstract}

\section{Introduction}

A thing is a concept that the gradual rise in recent years, which through various sensing devices and networks will be in the real world of people and things linked. Because each device has the ability to perceive the target, it is widely used for target localization and tracking fields. At this stage it made a lot of effective targeting and tracking algorithms, but due to the uncertainty of target motion, positioning and tracking accuracy has been greatly affected. To address this problem, we propose the idea of dividing the space, according to the location of each node is divided into different areas, packet tracing, the method using space piecewise linear approximation of thought to predict the target track, the target is lost when found, according to sensor network architecture proposed target recovery mechanisms. Simulation results show that the method used in this paper can effectively reduce network overhead, improve tracking efficiency.

\section{Research on issues related to targeting and tracking}

\section{Method of positioning.}

1) Three edge measurements

Three edge measurements is a known point by measuring the target point and the distance to determine the process of target position. If a beacon node and the target node can be obtained by measuring the distance between, so to beacon node as the center of the circle, the measured distance as the radius to draw the positioning round, each round is the goal location the intersection of node position, as shown in figure 1.

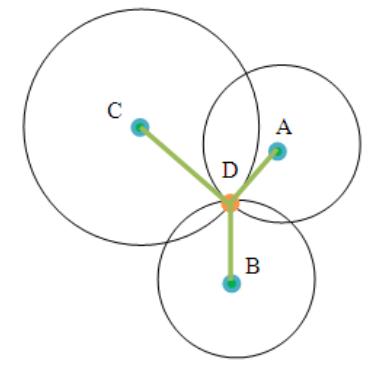

Fig.1 Three edge measurements 
2) Scene fingerprint orientation

Scene fingerprint positioning is mainly used to determine the signal of each node is receiving way, usually divided into two phases, as shown in figure 2.

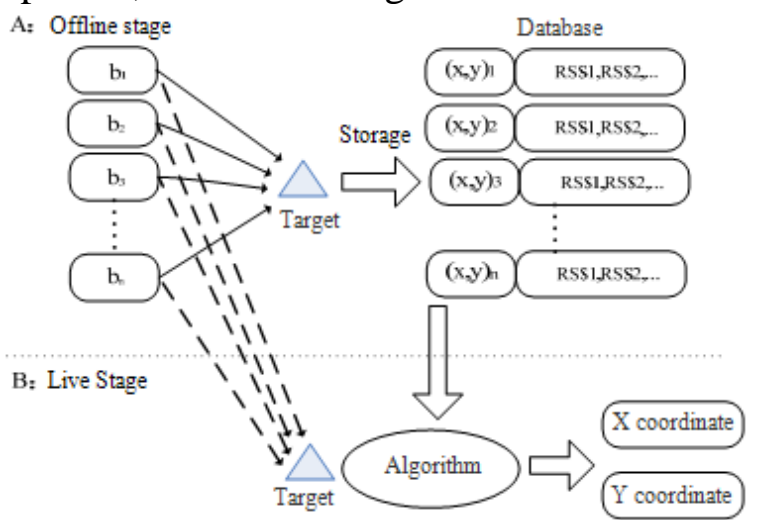

Fig.2 Scene fingerprint orientation method

Offline phase: measuring a moving target in different positions on the signal strength and the signal strength values collected and the corresponding position coordinates stored in the database.

Live Phase: a moving target from a collection of signal strength values within the range of the beacon signal is applied to the nodes, and then sends the information to the server, using the appropriate algorithm to estimate the position of the mobile object.

Tracking method.

1) Information driven collaborative tracking algorithm

Information driven collaborative tracking algorithm is a prediction mechanism based target tracking algorithm, the node to determine the direction of the target may according to own the detected information and the received information from other nodes, at the same time according to the prediction results to awaken the suitable nodes participating in the next moment tracking. In this way, the network always only a few nodes involved in the tracking task can save the energy to the maximum.

2) Delivery tree tracking algorithm

Transfer tree tracking algorithm uses a distributed approach, through local information exchange to complete tracking. When the goal is to capture the monitoring nodes to a later, through a certain election algorithm to select a root node, the root node in the tree structure of the transfer, to deliver messages to other nodes. Each node in the tree transmitted periodically sends tracking information back to the root node, the root node is responsible for data fusion, and sends the results to the sink. With a moving target, the transfer will remove those trees farther away from the target node; the new node is added simultaneously transmitted wake tree. Transmission tree tracking algorithm to ensure efficient tracking of the target, greatly reduce the communication cost, the principle shown in figure 3.

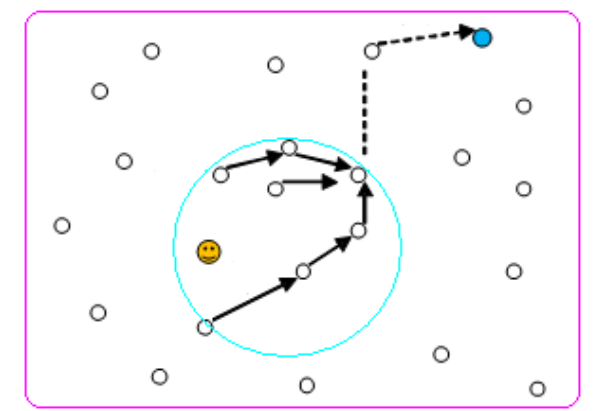

Fig.3 The principle of transfer tree tracking algorithm

\section{The Internet of things based on space partition target location and tracking algorithm}

Spatial clustering mechanism. Energy consumption is the target location and tracking algorithm is an important index, and reduce the energy consumption of one of the most effective way is to design efficient node coordination management mechanism. Only a handful of detected target nodes 
involved in tracking process, while the rest of the node to enter a dormant state, so that it can greatly reduce the system's overall energy consumption and prolong the network lifetime and thus, the resulting space clustering mechanism. Clustering mechanism is the basic idea is to partition in the network node, through the cluster head nodes within the section of information fusion and transmission between nodes and reduce the number of data transfer volume and distance, and thus reduce the energy consumption of communication, achieve the goal of network energy saving. In target tracking, each node according to the adjacent relation automatically organized into clusters, and then through a certain algorithm electing cluster heads. Cluster member responsible for tracking and task, the real-time collection of target location information, and is responsible for the collection of cluster nodes cluster members sent by monitoring target information, carry on the processing and fusion processing. Cluster heads in turn by each cluster member nodes randomly bear; in order to balance the energy consumption between clusters, prolong the lifecycle of the whole cluster.

Working mode. Spatial clustering algorithm in each node including three work modes: sleep mode and tracking mode and communication mode. Sleep mode: all modules are closed, only through their own awakening, timer 0 energy consumption; Most communication mode: the sleep state of the agreement, perception and other modules are closed, but communication module is in standby state, can be awakened the other nodes; Tracking mode: communication, perception and other modules are open. As shown in figure 4.

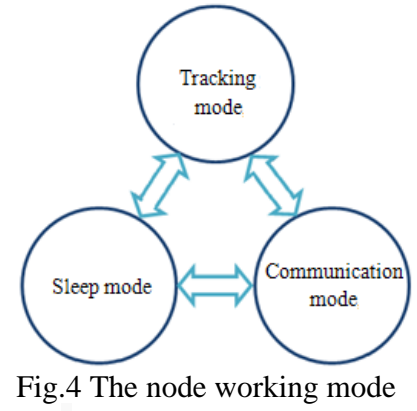

Target loss and recovery mechanism. Often moving target motion state is not fixed, movement direction, speed changes may occur suddenly, cause target from the current monitoring area. As shown in figure 5, the target node to predict the direction is right, but the actual direction into the lower right, lost this kind of situation is determined as the goal, to find the target again, the Internet of things based on space partition target localization and tracking algorithm is put forward the corresponding recovery mechanism.

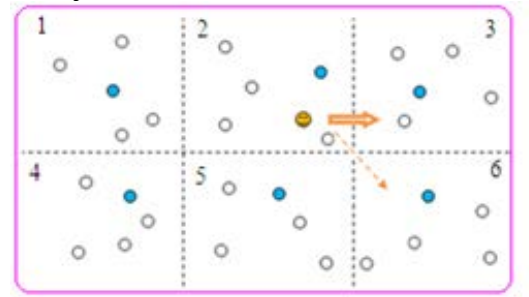

Fig. 5 Target lost

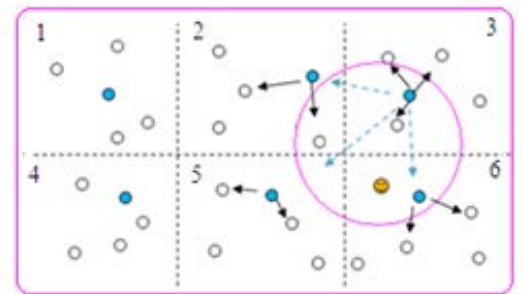

Fig.6 Target recovery

When target into 2 areas, according to track prediction target will enter 3 areas, 3 regional cluster head first launch detection, found the goal did not enter the area, the cluster head sends a signal to activate the surrounding scope, detect. 6 area cluster head found target at this moment, target, and will notify the other cluster heads dormancy, start tracking process, as shown in figure 6.

\section{Simulation Analysis}

In order to verify the performance of the target location and tracking algorithm, the tracking accuracy and energy consumption as the evaluation index, under the environment of Matlab simulation analysis. Simulation environment for the $100 \mathrm{~m}$ by $100 \mathrm{~m}$ x 100m cube space, 500 random deployments of sensor nodes in the area, each node communication radius are set to $50 \mathrm{~m}$, assuming that target mobile has no fixed pattern, moving speed and moving direction completely 
random. To test and verify the effectiveness of this algorithm, compared with the traditional method, the simulation results as shown in figure 7 , shown in figure 8.

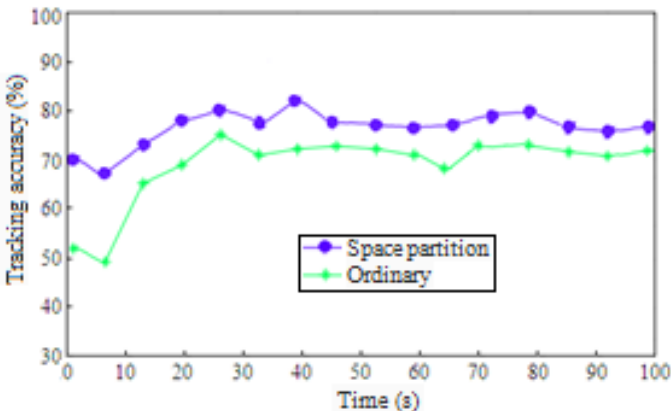

Fig.7 Tracking accuracy

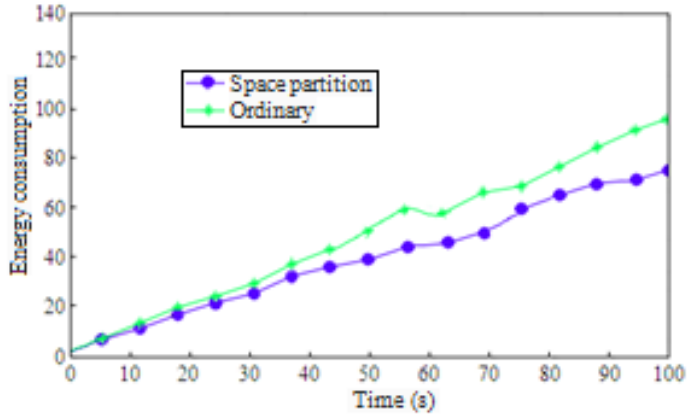

Fig.8 Energy consumption

Can be seen from the result, because of the space partition algorithm adopts the combined mechanism of cluster heads reduces the conversion that may occur during the process of target lost phenomenon, at the same time of improving positioning accuracy, improve the tracking accuracy, compared with the common algorithm, track the success rate increased by $10 \%$ or so. At the same time, because the target lost less, so relatively more common algorithms save energy consumption.

\section{Conclusion}

Target location and tracking technology is the basis of many applications, due to the randomness of the target motion state, elusive, bring difficulty positioning accuracy and tracking accuracy. In this paper, the target location and tracking method carried on the thorough analysis, and probes into the corresponding evaluation index; Proposes a iot target location and tracking based on space partition algorithm, the algorithm based on spatial clustering mechanism, to sleep, tracking and communication as the main working mode, the target for joint tracking. The simulation results show that compared with the common algorithm, this paper used algorithm has higher tracking accuracy and less energy consumption, to meet the design thought, to provide the reference to the research of localization and tracking technology.

\section{References}

[1] Bar-Shalom, F. Daum, J. Huang. The Probabilistic Data Association Filter [J]. IEEE Control Systems Magazine. 2009, 29(6): 82 100

[2] Y. W. Mo, D.Y. Xiao. Hybrid System Monitoring and Diagnosing Nosed on Particle Filter Algorithm. Acta Automation Sonica. 2003, 29(3): 641 648

[3] Vicaire P, He T, Cao Q, et al. Achieving long-term surveillance in Vigil Net[J] .ACM Transactions on Sensor Networks ( TOSN) , 2009, 5(1):1-39

[4] Z hai Y, Y eary B. A new centralized sensor fusion tracking methodology based on particle filtering for power aware systems. IEEE Transactions on Instrumentation and Measurement, 2008,57(10): 2377- 2387

[5] P. Bao. Localization Algorithm Based on Sector Scan for Mobile Wireless Sensor Networks [J]. Applied Mathematics \& Information Sciences. 2012, 6: 99-103 